\title{
La Literatura Científica en Ciencias Empresariales: un Análisis Comparativo entre Chile y Brasil
}

\author{
Patricio E. Ramírez ${ }^{(1)}$ y Ari M. Mariano(2) \\ (1) Escuela de Ciencias Empresariales, Universidad Católica del Norte, Larrondo 1281, Coquimbo-Chile. \\ (e-mail: patricio.ramirez@ucn.cl). \\ (2) Centro Universitario de Brasília, SEPN 707/907 - Asa Norte - Brasília - DF - Brasil. \\ (e-mail: ari.mariano@uniceub.br)
}

Recibido Jun. 13, 2014; Aceptado Ago. 21, 2014; Versión final recibida Ago. 26, 2014

\begin{abstract}
Resumen
Este estudio tiene por objetivo analizar la producción científica en el ámbito de las ciencias empresariales en Chile y Brasil. La metodología se basa en herramientas cienciométricas, usando como fuentes a 7955 artículos publicados en revistas indexadas Scopus entre 2004 y 2013. En resumen, los resultados indican que si bien en ambos países la producción científica ha aumentado en forma importante, se detecta una disminución del impacto de estos artículos. Además, existen diferencias entre Chile y Brasil asociadas a la cooperación con otros países, la variedad de las revistas donde se publican los estudios, y la estructura del ranking de las instituciones que publican. Las principales conclusiones indican que la investigación en este ámbito se realiza en universidades, la cooperación entre distintos países es significativa en esta producción científica, y la difusión se efectúa de una forma importante en revistas regionales.
\end{abstract}

Palabras clave: literatura científica, ciencias empresariales, investigación, publicaciones, Scopus

\section{Scientific Literature in Business Studies: A Comparative Analysis between Chile and Brazil}

\begin{abstract}
This study analyzes the scientific production in the field of business studies in Chile and Brazil. The methodology is based on scientometric tools, using as sources 7955 papers published in Scopus indexed journals between 2004 and 2013. In summary, the results indicate that although in both countries the scientific production has significantly increased, a reduction of the impact of these articles is detected. In addition, there are differences between Chile and Brazil associated to cooperation with other countries, the variety of journals where studies are published, and the structure of the ranking of the institutions that publish. The main conclusions indicate that research in this area is carried out in universities, cooperation between countries is significant in this scientific production, and dissemination is carried out mainly through regional journals.
\end{abstract}

Keywords: scientific literature, economics and business, research, publications, Scopus 


\section{INTRODUCCIÓN}

Ciencias empresariales como un área de las ciencias sociales cubre tópicos de negocios, gestión, contabilidad, economía, econometría, y finanzas. Si bien en un contexto global esta área ha ido creciendo y conformando un importante cuerpo de conocimientos (Díez et al., 2001), América Latina se ha caracterizado por una producción científica limitada en ella (Donoso y Crittenden, 2008). Dentro de América Latina, Brasil y Chile son considerados líderes en producción científica (Cano et al.,2012), y una importante característica de este desarrollo es estar fuertemente ligado a universidades (Ramírez y Alfaro, 2012; Leta et al., 2006), y no a centros de investigación como tales.

No obstante a lo anterior, y en particular en los casos de Chile y Brasil, el desarrollo económico sostenido y las iniciativas para aumentar el capital humano avanzado, dos variables asociadas positivamente (Álvarez et al., 2009), pueden estar revertiendo esta situación. De hecho, Chile y Brasil han incrementado su PIB entre 2000 y 2010 aproximadamente un 50\% (FMl, 2014), y ambos países poseen programas gubernamentales para desarrollar capital humano avanzado. Sin embargo, algunos autores indican que puede existir un freno a este avance debido a la baja inversión en investigación y desarrollo en relación a los líderes mundiales (Bitar, 2014). En este contexto, examinar las tendencias y resultados de la producción científica en ciencias empresariales en Chile y Brasil surge como un tema relevante para apoyar la gestión de incentivos y recursos dirigidos al colectivo de investigadores en el área.

Concordante con lo anterior, el objetivo de este estudio es analizar la producción científica en el ámbito de las ciencias empresariales en Chile y Brasil. En particular, basados en herramientas cienciométricas deseamos clarificar el avance de las publicaciones en ciencias empresariales durante la última década en estos dos países latinoamericanos. La originalidad de este estudio se basa en dos elementos, primero, la carencia de estudios que analicen las publicaciones en ciencias empresariales en Chile y Brasil, y segundo, la inexistencia de estudios comparativos entre Chile y Brasil en esta materia. Los siguientes apartados se han estructurado de la siguiente forma. Primero se explica el método utilizado en el estudio, para luego dar paso a los resultados del análisis, y finalmente a las conclusiones.

\section{MATERIALES Y MÉTODOS}

Este estudio se basa en métodos cienciométricos. Estos métodos utilizan los datos de las publicaciones científicas para analizar características de la investigación (Spinak, 2001). Si bien existen críticas asociadas a su uso (Bornmann y Daniel, 2008), estos métodos continúan prosperando (Bar-llan, 2008). La literatura muestra su utilidad, tanto para analizar áreas relacionadas a ciencias empresariales (Artto et al., 2009; Duque et al., 2006; Pratt et al., 2012), como para evaluar la producción científica de zonas geográficas (Abramo et al.,2008; Tan et al.,2010). Este estudio utiliza 1.470 artículos chilenos y 6.485 artículos brasileños, todos ellos indexados en la base de datos Scopus entre el año 2004 y diciembre de 2013. El análisis realizado busca responder las siguientes preguntas globales en relación a la investigación en ciencias empresariales en Chile y Brasil: a) ¿Cuántos artículos, en qué idioma y que impacto tienen?; b) ¿Dónde se publican estos artículos?; c) ¿Cuáles instituciones son las que más contribuyen?; y d) ¿Con que países se coopera en estas publicaciones?. Para poder evaluar la evolución del impacto de la publicaciones en el área se estableció un índice estandarizado de citas/publicaciones (IECP), calculado para cada año desde 2004 a 2013 según la ecuación 1:

$\operatorname{IECP}(\mathrm{x})=\frac{\mathrm{TC}(\mathrm{x})}{\mathrm{TP}(\mathrm{x})} * \mathrm{f}(\mathrm{x})$

Donde $x$ es el año, TC $(x)$ es el total de citas del año $x$ contabilizadas al 2013, TP ( $x$ ) es el total de publicaciones del año $x, y f(x)$ es un factor de ajuste de curva de citaciones del área, basado en trabajo de González-Pereira et al.(2010), y cuya lista de valores entre 2004 y 2013 es $\{25,23,21,18,16,13,10,7,4,1\}$. Para evaluar tendencias el estudio utiliza la prueba estadística no paramétrica de Cox y Stuart, asimismo, para evaluar variabilidad utiliza el coeficiente de variación.

\section{RESULTADOS}

La Tabla 1 muestra la cantidad de artículos de Chile y Brasil detallados por año, idioma, citas e índice estandarizado de citas/publicaciones. De estos resultados se deben destacar tres cosas. Primero, la clara tendencia al crecimiento de la cantidad de artículos, la prueba de Cox y Stuart indica que esta tendencia es estadísticamente significativa ( $p=0,01$ para Chile y Brasil). En efecto, entre 2004 y 2013 la cantidad de publicaciones se cuadruplicó en el caso de Chile y se octuplicó en el caso de Brasil. Segundo, si bien en ambos países el impacto de las publicaciones en inglés supera largamente al impacto en otros idiomas, su uso se ha conservado estable, la prueba de Cox y Stuart indica que no existe tendencia estadísticamente significativa ( $p=0,13$ en el caso de Chile y $p=0,45$ en el caso de Brasil). $Y$ tercero, existe una tendencia a la 
baja en el índice estandarizado del impacto de las publicaciones en ambos países, y esta tendencia es mucho más clara en el caso de Brasil. De hecho, en el caso de Brasil entre 2004 y 2012 este factor disminuyo un $75 \%$.

Tabla 1: Artículos chilenos y brasileños de ciencias empresariales

\begin{tabular}{|c|c|c|c|c|c|c|c|c|c|c|c|}
\hline Artículos & 2004 & 2005 & 2006 & 2007 & 2008 & 2009 & 2010 & 2011 & 2012 & 2013 & Total \\
\hline \multicolumn{12}{|c|}{ Chile } \\
\hline Número & 48 & 69 & 105 & 89 & 155 & 162 & 185 & 221 & 217 & 219 & 1470 \\
\hline - $\%$ Inglés & $68 \%$ & $78 \%$ & $91 \%$ & $89 \%$ & $76 \%$ & $63 \%$ & $75 \%$ & $73 \%$ & $76 \%$ & $84 \%$ & $77 \%$ \\
\hline - $\quad \%$ Español u otro & $32 \%$ & $22 \%$ & $9 \%$ & $11 \%$ & $24 \%$ & $37 \%$ & $25 \%$ & $27 \%$ & $24 \%$ & $16 \%$ & $23 \%$ \\
\hline Citas mayor igual a 1 & $79 \%$ & $82 \%$ & $77 \%$ & $78 \%$ & $76 \%$ & $59 \%$ & $59 \%$ & $49 \%$ & $36 \%$ & $19 \%$ & $54 \%$ \\
\hline - $\quad \%$ Inglés & $79 \%$ & $92 \%$ & $81 \%$ & $84 \%$ & $85 \%$ & $75 \%$ & $68 \%$ & $56 \%$ & $44 \%$ & $20 \%$ & $61 \%$ \\
\hline - $\quad \%$ Español u otro & $78 \%$ & $47 \%$ & $40 \%$ & $30 \%$ & $46 \%$ & $32 \%$ & $33 \%$ & $30 \%$ & $12 \%$ & $13 \%$ & $30 \%$ \\
\hline IECP & 0,26 & 0,57 & 0,39 & 0,55 & 0,35 & 0,27 & 0,30 & 0,22 & 0,25 & 0,26 & \\
\hline - Inglés & 0,34 & 0,71 & 0,42 & 0,62 & 0,44 & 0,40 & 0,38 & 0,27 & 0,32 & 0,28 & \\
\hline - Español u otro & 0,07 & 0,07 & 0,06 & 0,02 & 0,06 & 0,04 & 0,05 & 0,06 & 0,03 & 0,13 & \\
\hline \multicolumn{12}{|c|}{ Brasil } \\
\hline Número & 127 & 173 & 324 & 495 & 589 & 820 & 796 & 962 & 1150 & 1049 & 6485 \\
\hline - $\%$ Inglés & $88 \%$ & $90 \%$ & $66 \%$ & $68 \%$ & $59 \%$ & $63 \%$ & $65 \%$ & $69 \%$ & $66 \%$ & $68 \%$ & $67 \%$ \\
\hline - $\%$ Portugués u otro & $12 \%$ & $10 \%$ & $34 \%$ & $32 \%$ & $41 \%$ & $37 \%$ & $35 \%$ & $31 \%$ & $34 \%$ & $32 \%$ & $33 \%$ \\
\hline Citas mayor igual a 1 & $79 \%$ & $73 \%$ & $66 \%$ & $56 \%$ & $59 \%$ & $56 \%$ & $52 \%$ & $38 \%$ & $21 \%$ & $9 \%$ & $40 \%$ \\
\hline - $\%$ Inglés & $86 \%$ & $79 \%$ & $71 \%$ & $56 \%$ & $70 \%$ & $66 \%$ & $61 \%$ & $47 \%$ & $30 \%$ & $12 \%$ & $48 \%$ \\
\hline - $\%$ Portugués u otro & $27 \%$ & $24 \%$ & $57 \%$ & $54 \%$ & $43 \%$ & $40 \%$ & $33 \%$ & $19 \%$ & $4 \%$ & $2 \%$ & $25 \%$ \\
\hline IECP & 0,46 & 0,51 & 0,27 & 0,22 & 0,32 & 0,21 & 0,24 & 0,15 & 0,11 & 0,11 & \\
\hline - Inglés & 0,52 & 0,56 & 0,37 & 0,28 & 0,51 & 0,31 & 0,34 & 0,20 & 0,17 & 0,15 & \\
\hline - $\quad$ Portugués otro & 0,01 & 0,03 & 0,07 & 0,08 & 0,05 & 0,05 & 0,06 & 0,04 & 0,01 & 0,02 & \\
\hline
\end{tabular}

La Tabla 2 muestra las diez revistas donde se han publicado más artículos en ciencias empresariales de Chile y Brasil. En el caso de Chile, estás revistas concentran menos del $25 \%$ de las publicaciones, y en el caso de Brasil, menos del 36\%. Lo anterior indica la amplitud de las revistas utilizadas para difundir los resultados científicos en el área. Por otra parte, mayoritariamente las revistas más utilizadas para publicar aceptan aportaciones en idioma español, portugués e inglés.

Tabla 2: Ranking de revistas de publicaciones chilenas y brasileñas en ciencias empresariales

\begin{tabular}{|r|l|r|r|l|r|r|}
\hline \multirow{2}{*}{} & \multicolumn{2}{|c}{ Chile } & \multicolumn{2}{c|}{ Brasil } \\
\cline { 2 - 7 } & Revista & Artic. & \multicolumn{1}{c|}{$\%$} & Revista & Artic. & $\%$ \\
\hline 1 & Economía Chilena & 59 & $4,0 \%$ & Espacios & 411 & $6,3 \%$ \\
\hline 2 & Información Tecnológica & 49 & $3,3 \%$ & Gestao e Producao & 404 & $6,2 \%$ \\
\hline 3 & Latin American J. of Economics & 43 & $2,9 \%$ & Rev. Economia e Sociologia Rural & 273 & $4,2 \%$ \\
\hline 4 & Cepal Review & 35 & $2,4 \%$ & RAE Rev. Administracao Empresas & 233 & $3,6 \%$ \\
\hline 5 & Trimestre Económico & 34 & $2,3 \%$ & Revista De Economia Politica & 195 & $3,0 \%$ \\
\hline 6 & Estudios De Economia & 32 & $2,2 \%$ & Rev. Brasileira Gestao de Negocios & 173 & $2,7 \%$ \\
\hline 7 & Journal of Business Research & 29 & $2,0 \%$ & Economia Aplicada & 154 & $2,4 \%$ \\
\hline 8 & Resources Policy & 25 & $1,7 \%$ & Estudos Economicos & 152 & $2,3 \%$ \\
\hline 9 & Academia & 24 & $1,6 \%$ & J. Tech. Management\&Innovation & 148 & $2,3 \%$ \\
\hline 10 & Innovar & 24 & $1,6 \%$ & Revista Brasileira de Economia & 145 & $2,2 \%$ \\
\hline & Otras Fuentes & 1116 & $75,9 \%$ & Otras fuentes & 4197 & $64,7 \%$ \\
\hline
\end{tabular}

La Tablas 3 muestra las diez instituciones que más han publicado artículos en ciencias empresariales de Chile y Brasil. En su totalidad estas instituciones están ligadas a la formación universitaria. En el caso de Chile se destaca que las dos primeras instituciones triplican al menos los resultados de las otras ocho. En el caso de Brasil se destaca la primera institución que duplica al menos los resultados de las otras nueve. No obstante lo anterior, se observa una mayor homogeneidad de resultados de las instituciones brasileñas en relación a las chilenas (el coeficiente de variación en el caso de Brasil es 0,74 y en el caso de Chile 1,13). 
Tabla 3: Ranking de instituciones chilenas y brasileñas con artículos en ciencias empresariales

\begin{tabular}{|c|l|c|l|c|}
\hline \multirow{2}{*}{} & \multicolumn{2}{|c|}{ Chile } & \multicolumn{2}{c|}{ Brasil } \\
\cline { 2 - 5 } & Institución & Artículos & Institución & Artículos \\
\hline 1 & Universidad de Chile & 393 & Universidade de Sao Paulo & 1109 \\
\hline 2 & P. Universidad Católica de Chile & 328 & Fundação Getulio Vargas & 471 \\
\hline 3 & Universidad Adolfo Ibáñez & 104 & Universidade Federal do Rio de Janeiro & 399 \\
\hline 4 & Universidad de Concepcion & 69 & Universidade Federal do Rio Grande do Sul & 354 \\
\hline 5 & Universidad de Talca & 58 & Universidade de Brasilia & 253 \\
\hline 6 & Universidad Diego Portales & 52 & Universidade Federal de Santa Catarina & 246 \\
\hline 7 & Universidad del Desarrollo & 49 & Universidade Estadual de Campinas & 240 \\
\hline 8 & Universidad de Santiago de Chile & 41 & Universidade Federal de Minas Gerais & 233 \\
\hline 9 & Universidad Católica del Norte & 39 & P. Universidade Catolica do Rio de Janeiro & 222 \\
\hline 10 & Universidad del Bio Bio & 25 & Universidade Federal de Pernambuco & 182 \\
\hline
\end{tabular}

La Tabla 4 muestra los diez países con quienes más se ha cooperado en la elaboración de artículos en ciencias empresariales de Chile y Brasil. Es posible destacar tres elementos de este resultado. Primero, Estados Unidos se ubica en ambos países con el socio principal, sin embargo, en el caso de Chile la cooperación con ese país triplica el caso de Brasil. Segundo, la cooperación de Chile y España es destacada en esta área, situación que no se reproduce entre Brasil y Portugal, no obstante que estos dos últimos países comparten el idioma. Y tercero, el 3\% de las publicaciones chilenas en el área han cooperado con Brasil.

Tabla 4: Cooperación internacional en los artículos chilenos y brasileños en ciencias empresariales

\begin{tabular}{|c|l|r|c|r|}
\hline \multirow{2}{*}{} & \multicolumn{2}{|c|}{ Chile } & \multicolumn{2}{c|}{ Brasil } \\
\cline { 2 - 5 } & País de co-autores & $\%$ Artic. & País de co-autores & $\%$ Artic. \\
\hline 1 & Estados Unidos & $32 \%$ & Estados Unidos & $9 \%$ \\
\hline 2 & España & $12 \%$ & Reino Unido & $3 \%$ \\
\hline 3 & Reino Unido & $7 \%$ & Portugal & $2 \%$ \\
\hline 4 & Canada & $4 \%$ & Francia & $2 \%$ \\
\hline 5 & Francia & $4 \%$ & Canada & $2 \%$ \\
\hline 6 & Argentina & $3 \%$ & España & $1 \%$ \\
\hline 7 & Brasil & $3 \%$ & Alemania & $1 \%$ \\
\hline 8 & Alemania & $3 \%$ & Australia & $1 \%$ \\
\hline 9 & Australia & $3 \%$ & Países Bajos & $1 \%$ \\
\hline 10 & Italia & $2 \%$ & Italia & \\
\hline
\end{tabular}

\section{CONCLUSIONES}

El análisis de la producción científica en ciencias empresariales en Chile y Brasil entre 2004 y 2013 nos permite dar a continuación, a modo de conclusiones, respuestas globales a las preguntas que lo guiaron.

¿Cuántos artículos, en qué idioma y que impacto tienen? La producción científica en el área ha aumentado en forma importante, predominando el idioma inglés en su difusión, sin embargo se detecta una disminución del impacto de estos resultados. En definitiva y particularmente en el caso de Brasil, y consistente con Righetti (2013) y Novaes(2013), se ha aumentado en cantidad pero el impacto ha disminuido. Si asociamos este hallazgo con una relación aún baja en términos de inversión en investigación y desarrollo en relación a países de mayor producción científica (Bitar, 2014), podríamos interpretar que dado recursos financieros acotados, los investigadores están en un permanente conflicto entre la cantidad y el impacto de sus publicaciones.

¿Donde se publican? Si bien la difusión de la investigación en el área utiliza un amplio rango de revistas, esta se ha efectuado en un porcentaje importante en revistas regionales. Dado que calidad y visibilidad de las revistas son conceptos muy ligados, y sus problemas en Latinoamérica son motivo de preocupación desde hace décadas (Miguel, 2011), es posible que esta forma de difusión regional afecte negativamente el impacto de la producción científica en ciencias empresariales de Chile y Brasil. En el caso de Brasil, por una parte, las reconocidas dificultades para publicar en idioma extranjero de los investigadores en ciencias 
empresariales (Rosa y Alves, 2011), y por otra, la existencia de un sistema de calificación nacional que entrega pesos semejantes a revistas de prestigio académico desigual (Novaes, 2013), podrían causar la alta frecuencia de publicación en revistas locales. En el caso de Chile, la inclusión el año 2002 de la revistas chilenas indexadas en la base de datos SciELO al modelo de asignación del aporte fiscal directo a las universidades (Ramírez y Alfaro, 2012), puede ser un motivador importante para difundir resultados de investigación en revistas locales.

¿Cuáles instituciones son las que más contribuyen? Existe un limitado número de instituciones líderes en la producción científica en Chile y Brasil, y en general la investigación se realiza en universidades. Las entidades privadas no relacionadas con la educación son las grandes ausentes en la investigación en esta área, siendo esta realidad es rasgo característico y heredado de la investigación en general en Latinoamérica (Vaccarezza, 1998). En el caso de Brasil, observamos que las nueve universidades que más publican se asocian a los centros más desarrollados del país, esto es perfectamente explicable pues estos centros concentran un mayor número de investigadores e incentivos financieros (CNPQ, 2014). De hecho, el líder en número de doctores, el Sudeste de Brasil, duplica a la región que le sigue. En cuanto al financiamiento, por una parte, el Sudeste de Brasil concentra el mayor número de industrias que apoyan con recursos privados a la investigación, y por otra, dada la estructura de distribución de recursos del Estado según importancia, esta región también recibe los mayores aportes para investigación, ocasionando en un circulo de financiamiento y desarrollo de las mismas áreas e instituciones. Por ejemplo, la Universidad de São Paulo ha recibido más becas del Gobierno que las recibidas por el total de instituciones sumadas de 22 de las 26 provincias de Brasil (Moreno, 2014). En el caso de Chile, existe una clara centralización de las publicaciones en universidades de la región metropolitana, hecho consistente con la centralización en la mayoría de los ámbitos del país.

¿Con que países se coopera en estas publicaciones? La cooperación entre autores de distintos países es un elemento importante en la producción científica del área, destacando en ese aspecto Chile por sobre Brasil. De hecho y en los últimos años, en el caso de Chile este fenómeno se puede asociar al aumento global del porcentaje de colaboración internacional de los artículos en ciencias sociales (ver datos en http://www.productividad.informacioncientifica.cl). Sin embargo, resulta algo contradictorio que si bien Chile y Brasil son líderes latinoamericanos en investigación (Cano et al.,2012), la cooperación en ciencias empresariales entre autores de los dos países es muy baja.

Finalmente, dentro de las limitaciones de este estudio deseados destacar dos puntos. Primero, y en relación a la fuente de información del estudio, este trabajo se baso en la producción de artículos indexados en la base de datos Scopus, base de datos que si bien integra mayoritariamente la producción científica regional, es posible que no incluya revistas y/o libros donde algunos investigadores en ciencias empresariales difunden su trabajo. Y segundo, y en relación a la orientación del estudio, el enfoque cuantitativo seguido por los autores limita dar una reflexión global de la producción científica en ciencias empresariales, reflexión que creemos debería darse a la luz de trabajos cualitativos complementarios. Creemos que propuestas metodológicas para superar las dos anteriores limitaciones pueden orientan trabajos futuros.

\section{REFERENCIAS}

Abramo, G., D’Angelo, C. A., y Pugini, F., The measurement of Italian universities' research productivity by a non parametric-bibliometric methodology, Scientometrics, 76(2), 225-244 (2008).

Álvarez, A., Barraza, J. y Legato, A., Inversión Extranjera Directa y Crecimiento Económico en Latinoamérica, Información tecnológica, 20(6), 115-124 (2009).

Artto, K., Martinsuo, M., Gemünden, H. G. y Murtoaro, J., Foundations of program management: $A$ bibliometric view, International journal of project management, 27(1), 1-18 (2009).

Bar-llan, J., Informetrics at the beginning of the 21st century-A review, Journal of Informetrics, 2(1), 1-52 (2008).

Bitar, S., Las tendencias mundiales y el futuro de América Latina, Naciones Unidas, Santiago de Chile, (2014).

Bornmann, L. y Daniel, H., What do citation counts measure? A review of studies on citing behaviour, Journal of Documentation, 64(1), 45-80 (2008).

Cano, F., Harris, P., Schonhaut, L. y Ugarte, F., La producción científica en Chile y Latinoamérica, Revista chilena de pediatría, 83(1), 9-11 (2012).

CNPQ - Conselho Nacional de Desenvolvimento Científico e Tecnológico, Número de Doutores por região, disponible en http://estatico.cnpq.br/painelLattes/mapa/, acceso 5 de abril de 2014, (2013). 
Díez, E., García, J., Martín, F. y Periáñez, R, Administración y dirección, Editorial McGraw Hill, Madrid, (2001).

Donoso, P. y Crittenden, V. L., Strategic management in Latin America, Journal of Business Research, 61(6), 587-589 (2008).

Duque, E., Cervera, A. y Rodríguez, C., A bibliometric analysis of models measuring the concept of perceived quality inproviding internet service, Innovar, 16(28), 223-243 (2006).

FMI - Fondo Monetario Internacional Actualización de las perspectivas regionales: América Latina y el Caribe, disponible en http://www.imf.org/external/spanish/index.htm, acceso 1 de febrero de 2014, (2014).

González-Pereira, B., Guerrero-Bote, V. P. y Moya-Anegón, F., A new approach to the metric of journals' scientific prestige: The SJR indicator, Journal of informetrics, 4(3), 379-391 (2010).

Leta, J., Glänzel, W. y Thijs, B., Science in Brazil. Part 2: Sectoral and institutional research profiles, Scientometrics, 67(1), 87-105 (2006).

Miguel, S., Revistas y producción científica de América Latina y el Caribe: su visibilidad en SciELO, RedALyC y SCOPUS, Revista Interamericana de Bibliotecología, 34(2), 187-199 (2011).

Moreno, A., 90\% dos bolsistas do Ciência sem Fronteiras são de instituições públicas, Globo.com, disponible en http://g1.globo.com/educacao/noticia/2014/01/90-dos-bolsistas-do-ciencia-sem-fronteiras-saode-instituicoes-publicas.html, acceso el 5 de abril de 2014, (2014).

Novaes, W., A pesquisa em economia no Brasil: uma avaliação empírica dos conflitos entre quantidade e qualidade, Revista Brasileira de Economia, 62(4), 467-495 (2008).

Pratt, J. A., Hauser, K. y Sugimoto, C. R., Defining the intellectual structure of information systems and related college of business disciplines: a bibliometric analysis, Scientometrics, 93(2), 279-304 (2012).

Ramírez, P. y Alfaro, J., Desincentivo a la Investigación: Resultado del Comportamiento Inequitativo del Modelo de Aporte Fiscal Directo (AFD) a las Universidades Chilenas, Formación universitaria, 5(4), 2736 (2012).

Righetti, S., Brasil cresce em produção científica, mas índice de qualidade cai, Folha de S. Paulo: Ciência, (2013).

Rosa, A. y Alves, M., Pode o conhecimento em gestão e organização falar português?, RAE-Revista de Administração de Empresas, 51(3), 255-264 (2011).

Spinak, E., Indicadores cienciométricos, Acimed, 9, 16-18 (2001).

Tan, K., Goudarzlou, A. y Chakrabarty, A., A bibliometric analysis of service research from Asia, Managing Service Quality, 20(1), 89-101 (2010).

Vaccarezza, L., Ciencia, tecnología y sociedad: el estado de la cuestión en América Latina, Revista Iberoamericana de Educación, 18(1), 13-40 (1998). 